\title{
Sobre a definição de inovação em design: $O$ uso da análise de redes para explorar conceitos complexos
}

\author{
On the definition of innovation in design: Using \\ network analysis to explore complex concepts
}

Igor Reszka Pinheiro, Eugenio Andrés Díaz Merino, Leila Amaral Gontijo

inovação, design, análise de redes, análise de conteúdo innovation, design, network analysis, content analysis
Este artigo, com o objetivo de compreender as diferentes definições de inovação em design, mapeou todas as citações desse termo em um dos periódicos que melhor reflete o estado da arte das pesquisas desta área. O estudo exploratório, quantitativo e qualitativo foi realizado utilizando-se de uma variante das técnicas de análise de conteúdo e de análise de redes. Com base nos 13 artigos adequados encontrados, discerniu-se 163 categorias semânticas completamente conectadas em uma rede composta por oito clusters. De acordo com o quadro de referência compilado, a inovação em design aparentemente refere-se às alternativas empresariais socialmente aceitas para enfrentar os atuais problemas e os riscos futuros, especialmente através do desenvolvimento de novos produtos voltados para a satisfação das necessidades do mercado.

This paper, aiming at understanding the different definitions of innovation in the design domain, mapped all of its citations in one of the journals that best reflects its research state of art. This quantitative and qualitative exploratory study was conducted by using a variant of content analysis technique and the network analysis technique. Based on the 13 appropriate papers found, 163 semantic categories were discerned and fully connected in a network composed of 8 clusters. According to this framework, innovation in the design domain refers primarily to the socially accepted alternatives adopted by companies to tackle the present problems and the future risks, especially through the development of new tailor-made products to satisfy market needs.

\section{Introdução}

Poucas palavras são tão associadas ao campo do design quanto o termo inovação. Uma pesquisa no mecanismo de busca oferecido por inúmeras bases de dados científicos indica que a palavra inovação possui mais referências cruzadas com design que outros termos amplamente divulgados formal ou informalmente nessa mesma área, como estética, ergonomia, usabilidade, marca ou, mesmo, criatividade. Tamanha força de associação entre o design e a inovação se justifica por vários motivos. Primeiro, porque ambos atuam no sentido de fazer algo novo emergir propositalmente (Pinheiro, 2004). 
Segundo, porque ambos tem como fim o crescimento econômico, a capacidade de adaptação e a agregação de valor por meio de diferenciais competitivos (Design Council, 2011). Terceiro, porque o objeto de atuação de ambos os domínios são os processo, os serviços, as estruturas organizacionais e, sobretudo, os produtos (Chong \& Chen, 2010). Quarto, porque o principal meio empregado por ambos é a projeção de uma intenção no desconhecido (Nelson, Buisine \& Aoussat, 2013). E, finalmente, quinto, porque ambos se colocam no papel de intermediários entre as novas tecnologias e as necessidades dos usuários (Tezel, 2012).

A despeito da existência desse profundo entrelaçamento conceitual, o que se constata, porém, é que aparentemente nem os pesquisadores e nem os profissionais da área do design tem por hábito se engajar de maneira sistemática na tarefa de definir com precisão o termo inovação (D’Ippolito, 2014; Mutlu \& Er, 2003). Diferentemente do que ocorre em outros campos do conhecimento, pouco se investiga as particularidades da composição ou da manifestação da inovação no exercício efetivo do design. Tal realidade, que se constata sobretudo pela ausência de métricas bem definidas e amplamente aceitas para a sua aferição (Alves \& Bornia, 2011), além de impossibilitar o diálogo entre os resultados das diferentes linhas de pesquisa do campo, restringe o escopo das políticas públicas voltadas para o fomento da inovação por meio do design e, ainda, dificulta a adoção de métodos e técnicas projetuais mais eficazes para a difusão de suas criações no mercado.

O problema referente à definição da inovação em design, entretanto, não aparenta ser fruto da ausência de elaborações teóricas a respeito dessa temática, mas sim da sua tremenda profusão e divergência (Crossan \& Apaydin, 2010; D'Ippolito, 2014; Mutlu \& Er, 2003). Enquanto para o Manual de Oslo (OECD \& SOEC, 2005) a inovação em geral é definida como a implementação de novos ou significativamente melhores produtos, processos ou métodos de venda em mercados ou redes comerciais, os textos em design referem-se à inovação especificamente como o processo de desenvolvimento de novos produtos (Chong \& Chen, 2010), como uma etapa do ciclo de vida de um projeto (Baxter, 2000), como um instrumento de mudança social (Tezel, 2012) ou, mesmo, como o alinhamento estratégico de uma empresa com as demandas individuais da população (D’Ippolito, 2014). Nisso, não raro encontra-se uma miríade de adjetivos acompanhando o termo inovação, tais como radical, incremental, destrutiva, tecnológica, ambiental, ecológica, social, dentre outros, porém, não se faz referência alguma à forma com que todo esse conjunto de proposições se conecta.

Levando em consideração a importância da inovação para o desenvolvimento econômico das empresas e das nações (Jacobson, 1992; Greenacre, Gross \& Speirs, 2011; dentre outros), chegando a ser descrita como condição sine qua non para essas instituições se manterem em um ambiente concorrencial globalizado (Foster \& Kaplan, 2002), este artigo, então, tem como objetivo mapear 
graficamente todas as definições de inovação oferecidas pelo periódico com maior fator de impacto na área do design e, por conseguinte, explicitar o estado da arte desse processo nas pesquisas do campo. Tal proposta, mais que avançar de maneira sistemática na compreensão holística das múltiplas relações entre o design e a inovação, visa oferecer uma base semântica comum para a compilação de definições operacionais, o que se caracteriza como o primeiro passo rumo à criação de instrumentos uníssonos de medida da inovação em design.

Para tanto, este artigo possui a seguinte estrutura. A segunda seção descreve sucintamente o desenvolvimento da noção de inovação, a qual deriva originalmente dos antigos conceitos de imitação e de invenção. A terceira secção, por sua vez, descreve detalhadamente os procedimentos metodológicos empregados para rastrear e mapear a rede semântica do termo inovação, o que se realizou por meio da análise de conteúdo e da análise de redes. A quarta secção apresenta os resultados encontrados, tanto em termos numéricos quanto em termos gráficos, detalhando-se o grau de centralidade das palavras individuais e o conjunto de clusters mapeados como um todo. E, por fim, a quinta e a sexta secção discutem, respectivamente, os achados e as limitações desta pesquisa à luz de diferentes perspectivas adotadas por designers.

\section{Imitação, invenção e inovação}

É costume referir-se a J. Schumpeter como o pai da inovação e, destarte, como o primeiro expoente desse fenômeno (Godin, 20082012; Hospers, 2005). Apesar de ser verdade que em uma série de trabalhos (Schumpeter, 1912-1928-1947) ele, sozinho, revolucionou o campo da economia, mudando o seu foco do equilíbrio para a dinâmica dos mercados, não é sensato deixar de reconhecer aqueles que o precederam, sobretudo porque isso enviesaria a compreensão ontológica e epistemológica da própria inovação. Sendo assim, conforme sugere Godin (2008), esta revisão volta-se inicialmente para o entendimento da imitação e da invenção, conceitos que fundamentam a genealogia e, por conseguinte, a própria existência da inovação.

Uma vez que a tradição filosófica oriental concebe a própria realidade como uma entidade cíclica e harmônica, sem início e sem fim, as primeiras contribuições ontológicas no que diz respeito ao conceito de imitação, até onde os registros escritos permitem saber, estão presentes na cultura grega (Albert \& Runco, 1999). Enquanto para o dualista Platão a imitação consistia das imagens ou das demais sensações imperfeitas que os seres humanos são capazes de apreender sobre a verdade ou a realidade ideal dos deuses, para o monista Aristóteles a imitação referia-se às práticas que, de alguma forma, buscavam copiar ou reproduzir a natureza. Tais concepções, apesar de fundamentalmente distintas, atribuem valor ético e estético bastante similar ao ato da imitação, especialmente a artística, o qual 
era engrandecido conforme a qualidade da interpretação individual e dos artifícios empregados para a imposição da verdade ou da natureza ao meio artificial (Godin, 2008).

Neste período, a imitação, então, em nada era considera pejorativa, uma vez que ela possibilitava não somente o próprio aprendizado a respeito do mundo físico por meio da experimentação, mas também o barateamento dos bens de consumo, a aquisição de novas tecnologia alheias e, em geral, a difusão de boas práticas (Godin, 2008). Nisso, percebe-se que os critérios utilizados para se avaliar as imitações no decorrer da história não necessariamente dizem respeito à proximidade entre os objetos verdadeiros e seus representantes (fidedignidade), mas sim à capacidade que a mímica tem de sensibilizar um ou mais indivíduos, cujas opiniões passam a incentivar tal reprodução (utilidade). Destarte, a imitação, em certos aspectos, até hoje é indistinguível da inovação, já que ambas consistem da adoção e da posterior multiplicação de novas tendências sociais, sejam elas hábitos de consumo, técnicas expressivas, concepções políticas ou científicas, práticas culturais ou estratégias empresariais.

A diferença essencial que resta entre a inovação e a imitação, todavia, é a valorização do elemento individual que deu início à referida dinâmica social. Enquanto no conceito de inovação reconhece-se e valoriza-se o empreendedor como indivíduo essencial e, portanto, central no sistema social, no conceito de imitação relega-se ao reprodutor o papel de artífice geral, o qual é passível de substituição estrutural (Godin, 2008; Meheus \& Nickles, 1999). Surge, então, daqueles envolvidos em qualquer tipo de tarefa voltada para a mudança social (arte, ciência, política, comércio, guerra etc.), a necessidade de se proteger da indiferença cultural por meio da exaltação do elemento individual, o que ocorre, no século XIV, pela difusão do antigo termo invenção para designar as mesmas práticas interpretativas das até então chamadas de imitação (Godin, 2008-2012).

O termo invenção, originário da clássica arte da retórica, não pôde, porém, ser considerado um mero substituto da palavra imitação, pois, ao conferir identidade às forças motrizes da sociedade, ele adentrou no domínio do deus criador cristão. A palavra invenção, então, apesar de primordialmente referir-se à articulação de uma ideia, de um argumento ou de um fato para convencer ou persuadir os outros, enviesou-se para o simples ato de criação ex nihilo (De Masi, 2003). Nisso, a vertente monista predominante no contexto da imitação, este estruturado em um sistema social fechado, perdeu o seu lugar para a vertente dualista no contexto da invenção, uma vez que a própria concepção da absoluta novidade requer o acesso a outra dimensão da realidade. Tal perspectiva romântica da invenção perdura até os dias de hoje em algumas concepções a respeito da criatividade, as quais se sustentam em explicações de genialidade, inspiração, sorte ou dotação (Pinheiro \& Cruz, 2009).

Com o advento do empirismo de Francis Bacon no século XVII, a ciência, contudo, ganhou força por seu caráter utilitário 
e, finalmente, a prática de explicitar um determinado uso ou interpretação da natureza, inclusive nas artes e nas humanidades, passou a ser compreendida prioritariamente como uma questão de reconhecimento social (Albert \& Runco, 1999). Conforme Godin (2008), o marco que melhor sinaliza a transformação da ideia de invenção, da noção de divina criação para o reconhecimento de uma descoberta em um dado campo de atuação, é o início da concessão de patentes nominais aos novos produtores de tecnologias funcionais. Neste momento histórico, o termo invenção, mesmo afastado de suas concepções exotéricas, não se aproxima, porém, novamente da imitação, pois esta é tomada arbitrariamente como sua antítese no processo de registro legal.

A diferenciação entre invenções e imitações pode ser considerada arbitrária, não somente no sistema jurídico, mas também em qualquer outra área do conhecimento, sobretudo nas mais complexas, pois o critério último para a atestar a originalidade de um produto ou de uma ideia é o reconhecimento subjetivo de profissionais pares (Amabile, 1982; Csikszentmihalyi, 1996). Tal procedimento, mesmo obedecendo à rigorosa metodologia científica, ainda é limitado à expertise dos avaliadores, ao limiar de descontinuidade adotado por eles e, principalmente, aos seus interesses individuais (Nagel, 2001; Simonton, 1991). Desse modo, em última instância, o termo invenção caracteriza-se apenas como o objeto físico ou conceitual capaz de oferecer reconhecimento formal a um indivíduo que promove, de alguma forma, a descontinuidade em seu sistema social. Isso é coerente com o modelo de propulsão de Sternberg (1999), no qual um organismo individual ou coletivo qualquer pode alterar a inércia de seu sistema, basicamente, de sete diferentes maneiras: replicação; redefinição; incremento; incremento progressivo; redirecionamento; reconstrução e; reinicialização.

Tais mudanças sociais, mesmo sem possuírem igual formalização, foram justamente o objeto de estudo das primeiras teorias sociológicas e antropológicas de cunho evolucionista, incluindo a primeira teoria sobre a inovação, que é atribuída a Gabriel Tarde (Moldaschl, 2010). Tarde, como a maioria dos outros sociólogos do século XIX, não estava, porém, interessado no processo de inovação propriamente dito, mas sim no de difusão ou mudança cultural. Em seu trabalho, a inovação consistia apenas da proposição de novidades, as quais obtinham sucesso cultural na medida em que eram imitadas em detrimento de outras invenções concorrentes (Godin, 2008).

Desde o seu primeiro tratado, a inovação, então, é vista como uma força propulsora da sociedade, a qual emerge da capacidade individual de combinar ou interpretar, em tempo certo, diferentes elementos da natureza que passam a ser adotados, em função de sua utilidade, por todo um grupo de pessoas. Tal conceituação, apesar de bastante abrange, difere-se da imitação, cuja essência é a difusão social, e da invenção, cujo foco é a propulsão social, ao posicionar-se de maneira clara como a dialética existente entre o processo de invenção 
e o processo de imitação. Este terceiro processo, o de inovação, caracteriza-se, portanto, como o mediador entre a oferta individual e a demanda social, especialmente pelo recurso à eficiência empresarial (Gilbert \& Birnbaum-More, 1996).

Sobre esta base conceitual, iniciam-se os modelos da inovação desenvolvidos pelas ciências econômicas, de onde finalmente emerge a figura de J. Schumpeter. Segundo esse autor (1928), as invenções tecnológicas levavam às inovações empresariais, as quais, por sua vez, eram difundidas e geravam lucros reais. Ao contínuo e múltiplo processo de invenções, inovações e difusões foi dado o nome de "destruição criativa", uma vez que as novidades mais eficientes sempre ganham espaço no mercado pela queda de seus concorrentes (Foster \& Kaplan, 2002). Outros modelos posteriores ao seu, porém, propõem que a força motriz da inovação é a demanda social, o barateamento dos custos de produção, as tendências sociais amplas, a quantidade de informação dos consumidores, as alianças estratégicas ou, mesmo, a integração extensiva dos sistemas hierarquicamente superiores e inferiores de um empresa (Greenacre, Gross \& Speirs, 2011; Tidd, 2006).

A visão conjunta de todos os modelos da inovação permite a compreensão desse processo como a profunda integração de inúmeros atores, tanto internos quanto externos às empresas, com a intenção de gerar desenvolvimento econômico sustentável por toda a rede de relações (Tidd, 2006). O processo de inovação, então, por fim, torna-se conceitualmente um mecanismo de homeostase social que equilibra os interesses e as necessidades individuais através da sua reorganização estrutural, sempre que houver a percepção de mudanças ambientais. Para promover a integração entre a miríade de conceitos apresentados, a seguir descreve-se o método empregado para estruturar uma definição única e compreensiva da inovação no campo do design.

\section{Método}

Este estudo exploratório, de natureza quantitativa e qualitativa (Marconi \& Lakatos, 2007), foi realizado, basicamente, com auxílio de duas técnicas: uma variante da análise de conteúdo; e a análise de redes. Enquanto a primeira dessas técnicas tem como fim a definição e a contagem das categorias semânticas presentes em textos (Bardin, 1977), a segunda foi desenvolvida para compilar os atores ou as variáveis de um sistema e, assim, calcular as suas medidas de centralidade e agrupamento (Hanneman \& Riddle, 2005; Scott, 2000).

Os dados empregados nestas análises foram derivados exclusivamente do periódico Design Studies. Optou-se por esse lócus de pesquisa por três motivos: 1) porque o seu conteúdo abarca mais de trinta anos de investigações científicas na área do design; 2) porque o seu fator de impacto é o maior e, portanto, o mais representativo da área como todo; e 3) porque julga-se a qualidade dos seus artigos como excelente em padrões internacionais. Foram, então, selecionados para 
esta pesquisa todos os seus artigos que exibissem os termos innovation, innovative ou innovate nos campos título, resumo ou palavras-chave conforme procura realizada com ajuda do mecanismo de busca da base de dados ScienceDirect, disponíveis até março de 2014.

Os artigos encontrados foram lidos e todos os trechos textuais em que houve menção dos termos procurados foram tabelados. Excluiu-se da lista de citações os excertos em que a noção de inovação não era discutida ou adjetivada, aqueles em que a noção de inovação apresentada era plenamente redundante com outros trechos do mesmo artigo e aqueles em que a noção de inovação associava-se somente a exemplos de produtos ou processos pontuais. Além disso, refinou-se os trechos restantes para eliminar orações e apostos irrelevantes ao tema investigado, com base nos mesmos critérios de exclusão.

Cada trecho remanescente foi submetido à análise de conteúdo. Nesse procedimento, as palavras-chave de cada excerto foram discernidas (por exemplo, "Innovative design is defined as the improvement by extending the boundaries of particular precedents."; Al-kazzaz e Bridges, 2012), agregadas em um único glossário de termos (001 - improvement; 002 - extending; 003 - boundaries; 004 - precedents...) e, posteriormente, categorizadas em conjuntos de acordo com a sua afinidade semântica (por exemplo, different $\mathrm{E}$ unfamiliar $=007$ - different ). Essas categorias foram novamente associadas aos seus trechos originais, eliminando-se, assim, as duplicatas. Ressalta-se, então, que nesta pesquisa, diferentemente daquilo que ocorre no procedimento padrão da análise de conteúdo, não se computou os mesmos termos ou categorias semânticas duas ou mais vezes em um mesmo trecho. Ignorou-se tal contagem porque o objeto da análise de redes é o padrão de vínculos entre as variáveis, não a frequência de aparição desses elementos.

Nisso, as categorias semânticas foram cruzadas com base em seu pertencimento a um mesmo trecho textual. Em uma tabela quadrangular, cujas linhas e colunas identificam as mesmas categorias (001 - improvement; 002 - extending; [...] 095 - incremental; 096 resources...), atribuiu-se o valor 1 para os pares de categorias exibidos em um mesmo trecho (por exemplo, improvement e extending), e o para os demais pares (por exemplo, improvement e incremental). Tal procedimento, além de reproduzir o núcleo do conteúdo de cada trecho individual, possibilitou a conexão dos diferentes excertos por meio de suas categorias semânticas comuns. A matriz simétrica resultante foi inserida no software Ucinet 6 (Borgatti, Everett \& Freeman, 2002) e em seu programa auxiliar NetDraw (Borgatti, 2002) para os procedimentos da análise de redes.

No primeiro aplicativo investigou-se a semelhança estrutural das categorias semânticas, por meio de suas distâncias euclidianas (Burt, 1976), e calculou-se o nível de centralidade de cada elemento por meio dos seus autovalores (Bonacich, 1972). Dada a ausência de critérios de interrupção bem definidos para o procedimento de clusterização (Cheung \& Chan, 2005; Milligan \& Cooper, 1985), 
adotou-se exclusivamente como critério de corte a inspeção visual do gráfico formado pelos níveis de distanciamento euclidiano exibidos após aglutinação hierárquica de cada uma das categorias. Essa inspeção identificou o primeiro ponto de inflexão da Figura 1, momento em que a subdivisão da rede semântica geral já não aparenta contribuir significativamente para a sua compreensão. Já no segundo aplicativo, construiu-se o gráfico que mapeia a rede de relacionamentos semânticos investigada, através do procedimento iterativo padrão de escalonamento multidimencional métrico (iterative metric MDS) (Freeman, 2000; Scott, 2000). Os três resultados, por fim, foram compilados em uma única imagem para, dessa forma, tornar o mapa semântico da inovação mais inteligível.

Figura 1 Linha de inflexão presente nos níveis de clusterização. (Autor)

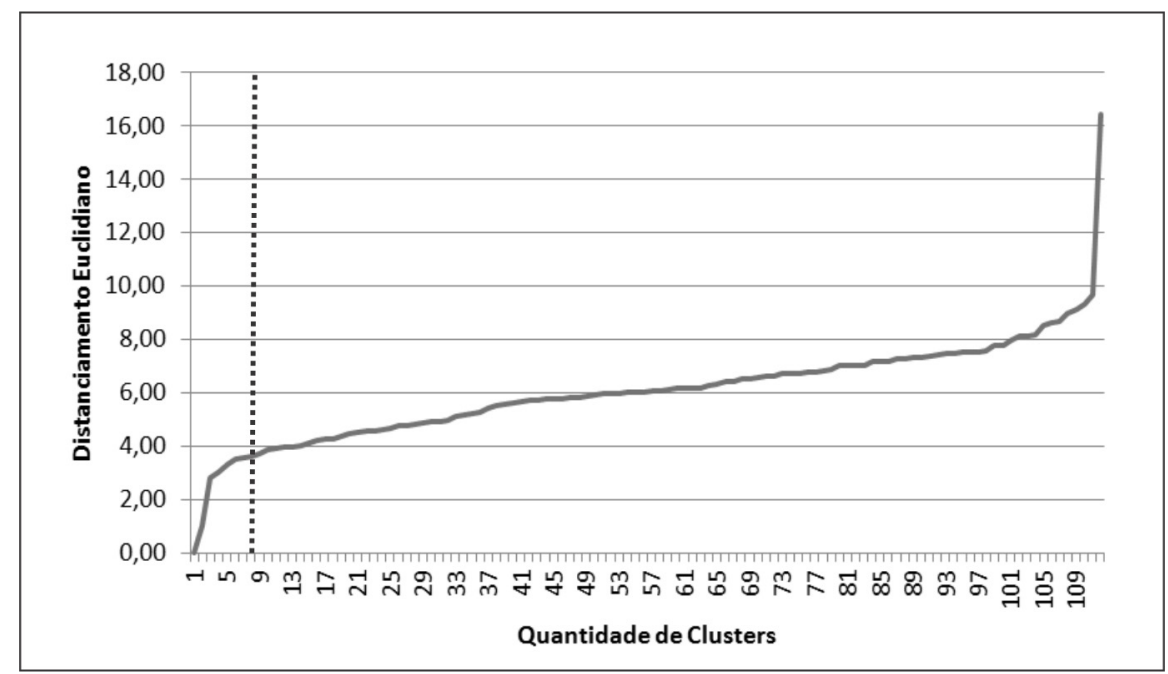

\section{Resultados}

1 Os artigo empregados como fonte de dados encontram-se marcas com um * nas referências

2 A listagem dos excertos é extensa demais para constar no artigo, mas eles encontram-se disponíveis para consulta junto ao primeiro autor deste artigo.
Com base nos parâmetros especificados, foram encontrados somente 13 artigos $^{1}$, os quais abrangem de forma bastante homogênea o período compreendido entre os anos de 1980 e 2012. Nenhum autor publicou mais de um artigo empregando os descritores afins à inovação, o que leva a crer a ausência de linhas de pesquisa dedicadas a essa temática no design, só projetos ou interesse isolados. Cada artigo, porém, forneceu em média 3,46 excertos ${ }^{2}$, o que indica uma elaboração não superficial do conceito de inovação de maneira individual. Como um todo, discerniu-se 163 categorias semânticas.

A análise das posições estruturais das categorias semânticas indica a presença de oito clusters coerentes entre si. O primeiro cluster é composto pelas categorias efficiency, environment, stakeholders, capture, brainstorming, user, interpretation e tactics. O segundo cluster é composto pelas categorias meaning, following, framing, mature, present, discipline, effect, value, prototype, first, knowledge, never, easy, artificial, affordance, symbolism, cultural, science, paradigm, service e 
communication. O terceiro cluster é composto pelas categorias change, problem, fostering, solution, objectives, potential, admittance, revelation, alignment, imagination e criterion. O quarto cluster é composto pelas categorias novelty, product, requirement, idea, market, technology, boundaries, success, wholeness, detail, stage, introduction, system, performance, principles, motivation, competition, regulation, advantage, evaluation, existence, development, creativity, demand, conflict, identifying, capability, people, process, implementation, radical, improvement, customers, creation, research, emergence, precedent, significant, experience, urge, particular, future e unsatisfaction. O quinto cluster é composto pelas categorias risk, decision, management, result, reconsideration, underlying, relationship, forecast e model. O sexto cluster é composto pelas categorias thinking, difference, practices, collaboration, appropriate, prevalence, sector, preference, resistance, insurance e climate. O sétimo cluster é composto pelas categorias concept, critical, characteristic, domain, combination, flexibility, functionality, broadening, component, persistence, enthusiasm, detachment, effort, independence, focus e social. E, por fim, o oitavo cluster, o maior de todos, é composto pelas categorias production, incremental, time, company, launch, beneficial, lead, structure, skills, interest, resources, world, tailoring, observation, extending, investment, alternative, complexity, application, renewal, economic, inspiration, manipulation, hard, work, practical, sort, entrepreneurial, pressure, inherence, increase, growth, rewards, attraction, costs, appearance, facilitation, means, transformation, uniqueness, strategy, project, exploitation e price.

Apesar do oitavo cluster ser o mais volumoso em termos de categorias semânticas, como pode ser observado na Tabela 1, o único cluster que exibe um nível de centralidade médio significativamente mais elevado que os outros, é o número $4(F(7)=46,10 ; p<0,001)$. Todas as dez categorias semânticas com o maior nível de centralidade da rede completa, aliás, figuram no mesmo cluster número 4 . Testes post hoc (Tukey) indicam que, além desse cluster estar em posição favorecida em relação a todos os outros, as únicas outras diferenças estatisticamente significativas referem-se à disposição mais periférica do cluster 2 em relação aos clusters número 3, 6, 7 e 8 . 
Tabela 1 Grau de centralidade das 10 categorias mais eminentes, dos clusters individualmente e da rede completa.

\begin{tabular}{|c|c|c|}
\hline Categoria semântica & Cluster & Centralidade \\
\hline Novelty / Novidade & 4 & 27,63 \\
\hline Product / Produto & 4 & 26,33 \\
\hline Requirement / Requisito & 4 & 25,28 \\
\hline Idea / Ideia & 4 & 23,70 \\
\hline Evaluation / Avaliação & 4 & 22,93 \\
\hline Market / Mercado & 4 & 22,66 \\
\hline Existence / Existência & 4 & 22,61 \\
\hline Technology / Tecnologia & 4 & 21,87 \\
\hline Development / Desenvolvimento & 4 & 21,81 \\
\hline Creativity / Criatividade & 4 & 20,41 \\
\hline Cluster 1 & & 3,55 \\
\hline Cluster 2 & & 2,07 \\
\hline Cluster 3 & & 8,73 \\
\hline Cluster 4 & & $17,21^{*}$ \\
\hline Cluster 5 & & 5,29 \\
\hline Cluster 6 & & 8,88 \\
\hline Cluster 7 & & 7,89 \\
\hline Cluster 8 & & 6,63 \\
\hline Rede Completa & & 9,03 \\
\hline
\end{tabular}

*Diferença estatisticamente significativa ao nível de 0,001

Nisso, observa-se que a rede formada pelo cruzamento de todas as categorias semânticas exibe um nítido grupo central, seis clusters intermediários e somente 1 cluster absolutamente periférico (Figura 02). Enquanto o cluster número 4, o núcleo da rede semântica da inovação em design, aparenta indicar a utilização do processo criativo (noção sintetizada com base nas categorias: creativity; creation; evaluation; development; conflict; identifying; process; experience; urge; insatisfaction) para o desenvolvimento de novos produtos capazes de redefinir o mercado (noção sintetizada com base nas categorias: novelty; product; idea; market; tecnology; boundaries; success; introduction; requirement; performance; competition; advantage; regulation), os clusters 3, 6 e 7 definem coletivamente uma dimensão de delimitação do tempo presente, e os clusters 1, 5 e 8, simetricamente, uma dimensão de previsão do tempo futuro. De forma individual, o cluster número 3 relaciona-se diretamente com a noção de problema (sintetizada com base nas categorias: problem; change; solution; admittance; objectives; criterion), o 
cluster 6 com a noção de cultura (sintetizada com base nas categorias: thinking; collaboration; climate; appropriate; practices; prevalence; difference; resistance) e o cluster 7 com a noção de funcionalidade (sintetizada com base nas categorias: functionality; characteristic; concept; critical; domain; component; persistence). Já o cluster número 1 reflete a noção de individualidade (sintetizada com base nas categorias: stakeholders; user; efficiency; interpretation; capture; brainstorming), o cluster 5 a noção de risco (sintetizada com base nas categorias: risk; decision; result; management; reconsideration; forecast; model) e o cluster 8 a noção de agilidade (sintetizada com base nas categorias: time; launch; lead; production; incremental; skills; renewal). O cluster número 2, por fim, aparenta configurar uma ampla dimensão de repercussões indiretas das inovações (meaning; science; prototype; value; effect; mature; knowledge; discipline; following; framing; symbolism).

Figura 2 Mapa semântico da inovação em design (Cluster 1: Triângulos-Padrão Cinza-Claro; Cluster 2: Losangos Brancos; Cluster 3: Quadrados Cinza-Escuro; Cluster 4: Círculos Brancos; Cluster 5: Quadrados Cinza-Claro; Cluster 6: Trinângulos-Invertidos Cinza-Escuro; Cluster 7: TriângulosPadrão Cinza-Escuro; Cluster 8: Triângulos-Invertidos Cinza-Claro). (Autor)

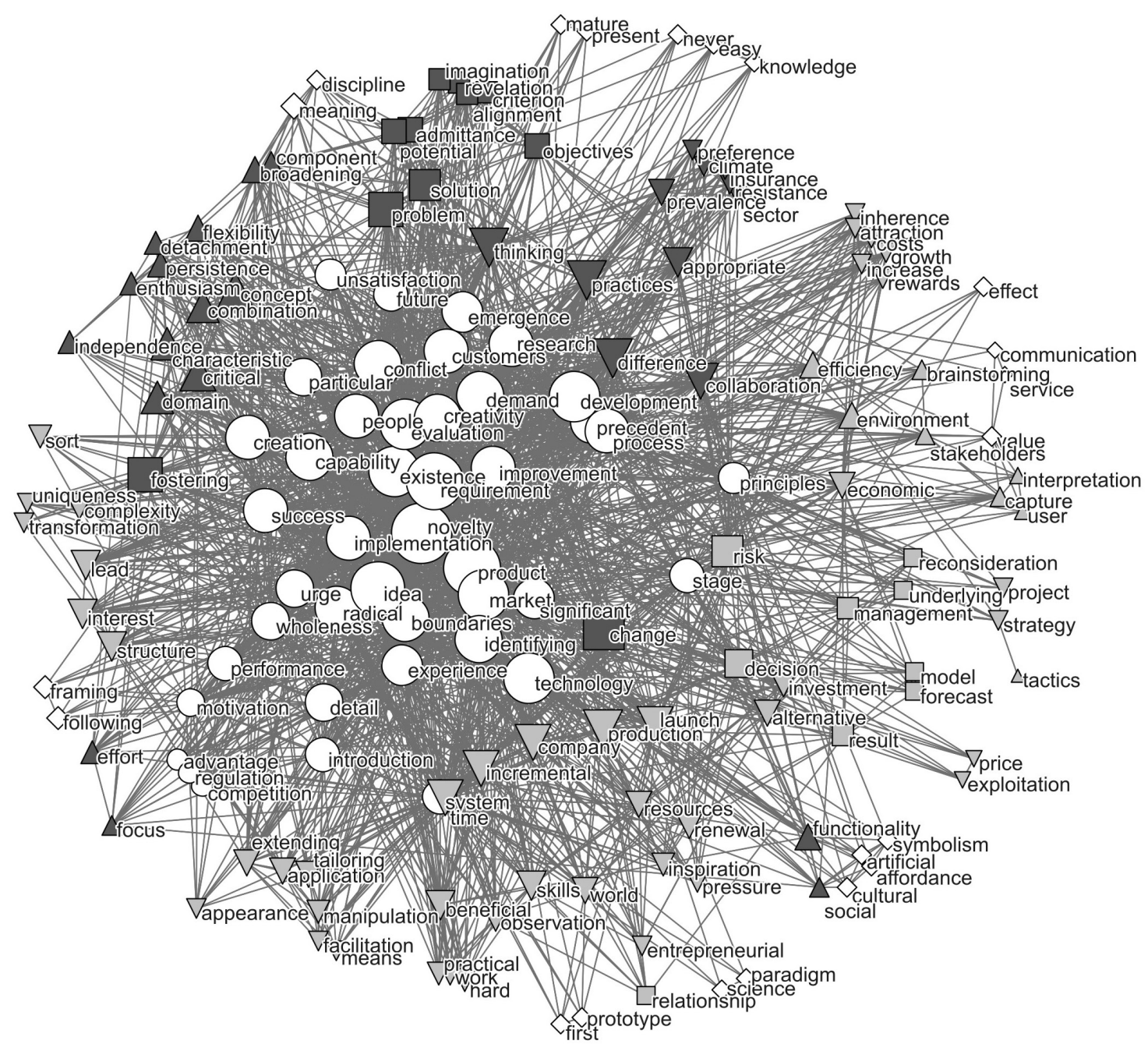


Destarte, obtém-se um mapa semântico do conceito de inovação em design, o qual ganha sentido não pela quantidade de dados compilados, mas sim pela estrutura subjacente discernida em seu conjunto de interelações. Na próxima secção discute-se tais conexões à luz das diferentes contribuições teóricas já apresentadas e busca-se definir um quadro de referência geral para a inovação em design.

\section{Discussão}

Como já era de se esperar, a definição de inovação em design é complexa. O que surpreende é o fato de que isso não significa, necessariamente, que ela é confusa, difusa ou, mesmo, profusa. Muito pelo contrário, apesar do pequeno número de artigos encontrados e da ausência de linhas de pesquisa nitidamente voltadas para o estudo da inovação em design, a rede semântica compilada com base nas diferentes abordagens sobre esse assunto está toda conectada, o que indica uma definição consideravelmente coesa e, por conseguinte, passível de compreensão comum. A dificuldade de se formalizar tal definição aparenta decorrer, então, mais do uso constante de recortes teóricos descontextualizados a respeito desse assunto, que da própria natureza do conceito de inovação em design.

Nisso, pode-se definir a inovação em design, com base no relacionamento encontrados entre os artigos pesquisados, basicamente como a alternativa intermediária adotada pelas empresas para enfrentar os problemas presentes e os riscos futuros, através do desenvolvimento de novos produtos direcionados para as necessidades do mercado (Figura 03). Define-se a inovação como uma alternativa intermediária, e não radical ou incremental, como ela muitas vezes costuma ser referida (Mutlu \& Er, 2003), porque ela exige métodos de produção que favoreçam, simultaneamente, a funcionalidade e a agilidade das empresas, bem como um foco, ao mesmo tempo, direcionado à cultura geral e aos interesses do consumidor individual. Enquanto o elemento que aparenta unir funcionalidade e agilidade é a criatividade projetual, aquele que aparenta unir cultura e indivíduo é a utilidade ou a eficiência do produto material. 
Figura 3 Quadro de referência geral da inovação em design. (Autor)

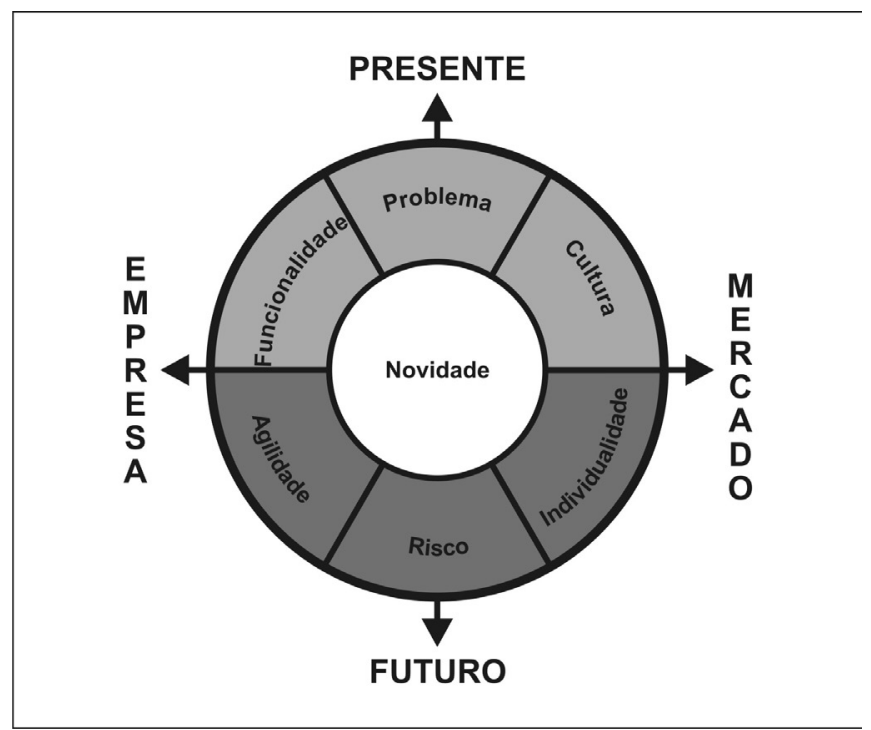

Percebe-se, então, que o movimento dialético entre invenção e imitação, bastante referido pelos campos da sociologia (Godin, 2008) e da economia (Kleijnen, Lee \& Wetzels, 2009), também está presente na definição de inovação em design. Para o design, as empresas são apontadas como a fonte de propulsão social e o mercado é considerado o principal mecanismo de difusão social. O detalhe que diferencia a perspectiva do design em relação à inovação é o fato de que, diferentemente do que se pressupõe nas demais áreas (Lane \& Maxfield, 2005), as invenções estão direcionadas para o enfrentamento não somente dos riscos do futuro, mas também dos problemas do presente. A definição de inovação em design sugere, portanto, que a novidade, advinda da criatividade, não é necessariamente uma forma de mover-se antecipadamente para o melhor cenário futuro, mas sim um atalho que conecta as diferentes capacidades empresariais às necessidades comerciais em ambas as direções temporais. Desse modo, é inovador empregar os recursos empresariais para lançar o presente ao futuro, elaborando novos produtos ou funcionalidades para conquistar ou revelar desconhecidos interesses individuais (novidade como atalho entre a funcionalidade e a individualidade na Figura 03), mas também é inovador aproximar o futuro do presente, agilizando a disponibilização de novos produtos para suprir as atuais demandas culturais (novidade como atalho entre a agilidade e a cultura na Figura 03).

Com base nessa perspectiva, pode-se dizer, então, que os designers utilizam a inovação como ponte de ligação entre os múltiplos interesses individuais e os diferentes interesses coletivos, o que caracteriza a ontologia sistêmica propagada pelas últimas gerações de modelos econômicos da inovação (Cecere, 2013). Essa corrente de pensamento, fundamentada na ideia de que a racionalidade das partes interessadas em qualquer transição comercial é limitada (Greenacre, 
Gross \& Speirs, 2011), sugere que a tomada de decisão individual sobre invenção ou imitação é indissociável da estrutura social em que esses mesmos sujeitos se encontram. Assim sendo, as inovações em design se definem essencialmente pelo impacto causado em seu contexto, de forma análoga aos diferentes movimentos inerciais propostos por Sternberg (1999).

Em termos de aferição do impacto da inovação em design, compreende-se, portanto, que a mais provável possibilidade de caracterização do seu sucesso inicia-se com a contextualização, ou seja, a relativização, dos diferentes atores sociais interessados no projeto (Audretsch, 1995). Por um lado, uma inovação só é bem sucedida se for capaz de conciliar os interesses dos vários profissionais de uma mesma organização em relação às práticas de imitar e de inventar, pois, do contrário, haverá divergência interna e consequente perda de capital social (Dylag et al., 2013). Por outro, uma inovação também só é bem sucedida se for capaz de conciliar os interesses dos inúmeros indivíduos de uma mesma sociedade em relação às práticas de imitar e de inventar, pois, do contrário, haverá resistência externa e consequente queda dos lucros (Kleijnen, Lee \& Wetzels, 2009). As métricas voltadas para a avaliação da inovação em design devem, então, favorecer as propostas em que as contribuições individuais são mais facilmente reconhecidas como relevantes e úteis ao seu contexto social. Produtos, projetos ou ideias são inovadores na medida em que mesmo os grupos de interesses divergentes, internamente em uma empresa ou externamente na sociedade, reconhecem o seu valor e os aceitam como contribuições válidas à sua estrutura social.

A primazia do termo inovação nos textos sobre design marca, destarte, o espírito de uma época em que a principal tarefa assumida por esses profissionais é a conciliação dos interesses individuais com os interesses coletivos, por meio da criação de novos produtos com maior valor. Tal proposição alinha de maneira bastante clara os resultados encontrados nesta pesquisa com a posição de Bertola e Teixeira (2003) e de Robinson e colaboradores (2005), para os quais atualmente o designer é sobretudo um agente do conhecimento, este inserido nas organizações para promover a integração interna, para desvendar necessidades ou oportunidades externas, para liderar mudanças e para implementar sistemas produtivos de alto valor agregado. O núcleo dessas atividades é, muito provavelmente, promover a compreensão conjunta dos requisitos e do benefícios de um projeto qualquer (Kleinsmann \& Valkenburg, 2008).

Nisso, o campo do design, dentro das limitações desta pesquisa, entende a inovação como um meio de obtenção de lucros reais sem que haja a necessidade de sacrifícios individuais. Para tal, com base no quadro de referência geral da inovação aqui proposto (Figura 03), especula-se que seja necessário o equilíbrio dinâmico entre as forças endógenas e exógenas do sistema social em que as empresas se inserem, pois o critério definitivo para o favorecimento da imitação ou da invenção em um projeto qualquer é a vontade ou a 
capacidade dos indivíduos de resistirem às normas culturais para a obtenção dos novos produtos materiais. A novidade só ganha espaço empresarial quando ela é eficiente na tarefa de favorecer o bemestar dos indivíduos em seu meio, ou seja, quando ela promove a homeostase social.

\section{Considerações finais}

Este artigo buscou definir o termo inovação em design com base em seu uso corrente pelos profissionais ou pesquisadores da área, e não por suas origens etimológicas. Até onde vai o conhecimento dos autores, esta é a primeira tentativa sistemática de realizar tal definição por meio de uma fusão das técnicas de análise de conteúdo e análise de redes. Os resultados dessa iniciativa apontam para a definição de que a inovação em design é, sucintamente, a alternativa intermediária adotada pelas empresas para enfrentar os problemas presentes e os riscos futuros, através do desenvolvimento de novos produtos direcionados para as necessidades do mercado. Com isso em mente os projetistas podem direcionar melhor os esforços de venda e inserção do próprio design no processo de inovação geral dos países e do mundo.

Tal definição deve, contudo, ser interpretada dentro das limitações deste estudo. Primeiro, reconhece-se que apesar do periódico Design Studies ser representativo de sua área como um todo, ele ainda é apenas uma dentre as várias possíveis fontes de dados existentes em relação à inovação em design. Segundo, a análise de conteúdo, por mais criteriosa que seja a sua condução, sempre consistirá de uma redução de dados através de sua categorização, o que implica um viés de interpretação. Terceiro, o procedimento de clusterização da análise de redes também depende de um ponto de corte subjetivo. Toda alteração realizada em alguma dessas variáveis poderia acarretar uma nova configuração estrutural das categorias semânticas e, portanto, uma definição diferente de alguns aspectos da inovação em design.

Sendo assim, recomenda-se que os interessados nesta linha de pesquisa busquem superar essas limitações ou, mesmo, replicar os achados aqui apresentados utilizando outras fontes de dados. Além disso, futuras pesquisas vinculando a inovação ao design também podem voltar-se para as tarefas de desenvolver medidas das contribuições individuais em meio ao seu contexto social, de adaptar técnicas projetuais ao caráter dialético do processo de inovação ou, ainda, de divulgar os achados desta pesquisa para o grande público, uma vez que a aceitação social do quadro de referência aqui proposto é um caminho para a formalização de políticas públicas voltadas para o fomento da inovação através do design. Enquanto nenhum desses avanços é dado, espera-se que este artigo, pelo menos, sirva como base para a discussão sistemática da definição da inovação em design, pois 
afinal, julga-se que o meio mais produtivo de se abordar um assunto rico em detalhes, divergências ou, mesmo, contradições, é visualizar aquilo que há em comum em todas as partes.

\section{Referências}

ALBERT, R. \& RUNCO, M. 1999. A history of research on creativity. In: Sternberg, R. (Ed.) Handbook of creativity: 16-31. New York: Cambridge University Press.

*AL-KAZZAZ, D. \& BRIDGES, A. 2012. A framework for adaptation in shape grammars. Design Studies, v.33: 342-356.

ALVES, L. \& BORNIA, A. 2011. Desenvolvimento de uma escala para medir o potencial empreendedor utilizando a Teoria da Resposta ao Item (TRI). Gestão \& Produção, v.18, n.4: 775-790.

AMABILE, T. 1982. Social psychology of creativity: a consensual assessment technique. Journal of Personality and Social Psychology, v.43, n.5: 997-1013.

AUDRETSCH, D. 1995. Firm profitability, growth, and innovation. Review of Industrial Organization, v.10: 579-588.

BARDIN, L. 1977. Análise de conteúdo. Lisboa, Portugal: Edições 70.

BAXTER, M. 2000. Projeto de produto: guia prático para o design de novos produtos. São Paulo: Edgard Blücher.

*BERTOLA, P., \& TEIXEIRA, J. 2003. Design as a knowledge agent: how design as a knowledge process is embedded into organizations to foster innovation. Design Studies, v.24: 181-194.

BONACICH, P. 1972. Factoring and weighting approaches to status scores and clique identification. Journal of Mathematical Sociology, v.2: 113-120.

BORGATTI, S. 2002. Netdraw network visualization. Harvard, MA: Analytic Technologies.

BORGATTI, S.; EVERETT, M. \& FREEMAN, L. 2002. Ucinet for Windows: software for social network analysis. Harvard, MA: Analytic Technologies.

BURT, R. 1976. Positions in networks. Social Forces, v.55: 93-122.

*CANDI, M. \& SAEMUNDSSON, R. 2008. How different? Comparing the use of design in service innovation in Nordic and American new technology-based firms. Design Studies, v.29: 478-499.

CECERE, G. 2013. The economics of innovation: a review article. Journal of Technological Transformation, October.

CHEUNG, M. \& CHAN, W. 2005. Classifyng correlation matrices into relatively homogeneous subgroups: a cluster analytic approach. Educational and Psychological Measurement, v.65, n.6: 954-979.

CHONG, Y. \& CHEN, C. 2010. Customer needs as moving targets of product development: a review. International Journal of Advanced Manufacturing Technology, v.48: 395-406.

*CROSS, N. \& CROSS, A. 1996.Winning by design: the methods of Gordon Murray, racing car designer. Design Studies, v.17: 91-107.

CROSSAN, M. \& APAYDIN, M. 2010. A multi-dimensional framework of organizational innovation: a systematic review of the literature. Journal of Management Studies, v.47, n.6: 1154-1191. 
CSIKSZENTMIHALYI, M. 1996. Creativity: flow and the psychology of discovery and invention. Nova Iorque: Harper Perenial.

DE MASI, D. 2003. Criatividade e grupos criativos. Rio de Janeiro: Sextante.

DESIGN COUNCIL. 2011. Design for innovation: facts, figures and practical plans for growth. Design Council. Disponível em: www.designcouncil.org.uk. Recuperado em: 01 de agosto de 2013.

D'IPPOLITO, B. 2014. The importance of design for firms' competitiveness: a review of the literature. Technovation, prelo.

DYLAG, A., et al. 2013. Discrepancy between individual and organizational values: occupational burnout and work engagement among white-collar workers. International Journal of Industrial Ergonomics, v.43: 225-231.

FOSTER, R. \& KAPLAN, S. 2002. Destruição criativa. Rio de Janeiro: Campus.

FREEMAN, L. 200o. Visualizing social networks. Journal of Social Structure, v.1, n.1.

GARDINER, P. \& ROTHWELL, R. 1985. Tough costumers: good designs. Design Studies, v.6, n.1: 7-17.

GILBERT, J. \& BIRNBAUM-MORE, P. 1996. Innovation timing advantages': from economic theory to strategic application. Journal of Engineering and Technological Management, v.12: 245-266.

GODIN, B. 2012. "Innovation studies": the invention of a specialty. Minerva, v.50: $397-421$.

GODIN, B. 2008. Innovation: the history of a category. Working Paper No. 1, Project on the Intellectual History of Innovation, Montreal, Canada.

GREENACRE, P.; GROSS, R. \& SPEIRS, J. 2011. Innovation theory: a review of the literature. ICEPT Working Paper, Imperial College, London.

HANNEMAN, R. \& RIDDLE, M. 2005. Introduction to social network methods. Riverside, CA: University of California.

HOSPERS, G. 2005. Joseph Schumpeter and his legacy in innovation studies. Knowledge, Technology, \& Policy, v.18, n.3: 20-37.

JACOBSON, R. 1992. The "Austrian" school of strategy. Academy of Management Review, v.17: 782-807.

JONES, E.; STANTON, N. \& HARRISON, D. 2001. Applying structured methods to Ecoinnovation: an evaluation of the Product Ideas Tree diagram. Design Studies, v.22: 519-542.

KLEINSMANN, M. \& VALKENBURG, R. 2008. Barriers and enablers for creating shared understanding in co-design projects. Design Studies, v.29: 369-386.

KLEIJNEN, M.; LEE, N. \& WETZELS, M. 2009. An exploration of consumer resistance to innovation and its antecedents. Journal of Economic Psychology, v.30: $344-357$.

LANE, D. \& MAXfiELD, R. 2005. Ontological uncertainty and innovation. Journal of Evolutionary Economics, v.15: 3-50.

LEHOUX, P.; HIVON, M.; WILLIAMS-JONES, B. \& URBACH, D. 2011. The worlds and modalities of engagement of design participants: a qualitative case study of three medical innovations. Design Studies, v.32: 313-332.

LITTLE, S. 1987. Incremental and systematic innovation strategies: reflections of technical choice. Design Studies, v.8, n.1: 41-54.

LITTLER, D. 1980. Perspectives on in-company technological innovation. Design Studies, v.1, n.6: 349-352. 
MARCONI, M. \& LAKATOS, E. 2007. Fundamentos de metodologia científica. São Paulo: Atlas.

MEHEUS, J. \& NICKLES, T. 1999. The methodological study of creativity and discovery: some background. Foundations of Scienve, v.4: 231-235.

MILLIGAN, G. \& COOPER, M. 1985. An examination of procedures for determining the number of clusters in a data set. Psychometrika, v.50, n.2: 159-179.

MOLDASCHL, M. 2010. Why innovation theories make no sense. Papers and reprints of the department of innovation research and sustainable resource management, Chemnitz University of Technology.

MUTLU, B. \& ER, A. 2003. Design innovation: historical and theoretical perspectives on product innovation by design. Proceedings of the $V$ European Academy of Design Conference, Barcelona, Spain.

NAGEL, S. 2001. Measuring creativity. The Innovation Journal. Disponível em: http:// www.innovation.cc/index.html . Recuperado em: 03 de novembro de 2007.

NELSON, J.; BUISINE, S. \& AOUSSAT, A. 2013. Anticipating the use of future things: towards a framework for prospective use analysis in innovation design projects. Applied Ergonomics, v.44: 948-956.

ORGANIZATION FOR ECONOMIC CO-OPERATION AND DEVELOPMENT; STATISTICAL OFFICE OF THE EUROPEAN COMMUNITIES. 2005. Oslo Manual: guidelines for collecting and interpreting innovation data (3a ed). Paris: Organisation for Economic Co-operation and Development.

PETRE, M. 2004. How expert engineering teams use disciplines of innovation. Design Studies, v.25: 477-493.

PINHEIRO, I. 2004. MCD - Método Criativo em Design: uma proposta com base nas áreas de inteligência artificial, psicologia, metodologia de projetos e criação de produtos. Estudos em Design, v.12 n.2: 37-51.

PINHEIRO, I. \& CRUZ, R. 2009. Fundamentos históricos e epistemológicos da pesquisa objetiva em criatividade. Psico, v.40, n.4: 498-507.

ROBINSON, M.; SPARROW, P.; CLEGG, C. \& BIRDI, K. 2005. Design engineering competencies: future requirements and predicted changes in the forthcoming decade. Design Studies, v.26: 123-153.

ROY, R. 1993. Case studies of creativity in innovative product development. Design Studies, v.14, n.4: 423-443.

SCHUMPETER, J. 1947. The creative response in economic history. Journal of Economic History, November: 149-159.

SCHUMPETER, J. 1928. The instability of capitalism. The Economic Journal, September: 361-386.

SCHUMPETER, J. 1912. The theory of economic development: an inquiry into profits, capital, credit, interest, and the business cycle. Cambridge: Harvard University Press.

SCOTT, J. 2000. Social network analysis: a handbook. Thousand Oaks, CA: Sage Publications.

SIMONTON, D. 1991. Latent-variable models of posthumous reputation: a quest for Galton's g. Journal of Personality and Social Psychology, v.6o, n.4: 607-619.

SMITH, S.; SMITH, G. \& SHEN, Y. 2012. Redesign for product innovation. Design Studies, v.33: 160-184.

STERNBERG, R. 1999. A propulsion model of types of creative contributions. Review of General Psychology, v.3, n.2: 83-100. 
TEZEL, E. 2012. Theoretical and historical perspectives in design, innovation and policies. Proceedings of the II International Conference of Mukogawa Women's University, Japan.

TIDD, J. 2006. A review of innovation models. Working Paper, Imperial College, London, 1-16.

\section{Sobre os Autores}

\section{Igor Reszka Pinheiro,}

Dr. UFSC / Unifebe, Brasil <pinheiro_ir@yahoo.com.br>

\section{Eugenio Andrés Díaz Merino,}

Dr. UFSC, Brasil <eugenio.merino@ufsc.br>

Leila Amaral Gontijo,

Dra. Université de Paris XIII / UFSC, Brasil <leila.gontijo@ufsc.br>

Artigo recebido em 06 mai. 2015, aprovado em 10 dez. 2015. 